\title{
COVID-19 y coberturas de vacunación del calendario regular del Paraguay, efecto de la pandemia
}

\author{
COVID-19 and Vaccination Coverage of the Regular \\ Calendar of Paraguay, effect of the Pandemic
}

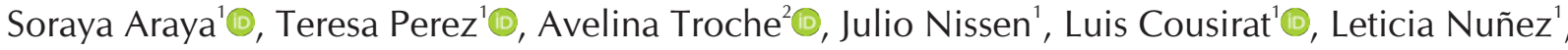

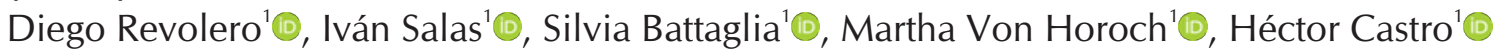 \\ ${ }^{1}$ Ministerio de Salud Pública y Bienestar Social, Programa Ampliado de Inmunizaciones. Asunción, \\ Paraguay. \\ ${ }^{2}$ Instituto de Medicina Tropical. Asunción, Paraguay.
}

\section{RESUMEN}

Introducción: La pandemia de COVID-19 produjo una crisis sanitaria afectando las coberturas de vacunación de los países. Objetivo: Describir la cobertura de vacunación de los biológicos trazadores durante el periodo prepandémico (2015- 2019) y pandémico (2020-2021) en Paraguay. Materiales y Métodos: Estudio descriptivo observacional de corte transverso, que comprendió a niños de 0 a 5 años de edad, de las 18 Regiones Sanitarias del País. Se analizaron las coberturas de vacunación del Programa Ampliado de Inmunización, periodo prepandémico (2015-2019) y pandémico (2020-201). Se incluyeron los biológicos trazadores: BCG, DPT1, DPT3, IPV1 y bOPV3, SPR1, SPR2 y vacuna antiamarílica (AA), el cálculo de cobertura de vacunación a nivel país se realizó por medio del análisis del reporte electrónico semanal de dosis de vacunas administradas por Región Sanitaria. Se estableció la comparación de las coberturas de vacunación por biológico trazador, por periodos y rango de edad. Resultados: Durante la pandemia se constató un descenso de cobertura de vacunación de los biológicos trazadores, para BCG: $4 \%$ (2020), 15\% (2021);DPT1: $5 \%$ (2020), $13 \%$ (2021); DPT3: $9 \%$ (2020), $22 \%(2021)$; IPV1: $5 \%$ (2020), $16 \%$ (2021); bOPV3: 7\% (2020),19\% (2021); SPR1: $9 \%$, SPR2: 13 \% (2020),SPR1:17\%, SPR2:16 \% (2021) у AA: 7\% (2020), 15\% (2021). Conclusión: Durante la pandemia de Covid19 disminuyeron las coberturas de vacunación de todos los biológicos trazadores, similar descenso se constató en otros países de América, existe el riesgo de aparición de

\section{ABSTRACT}

Introduction: The COVID-19 pandemic produced a health crisis affecting countries' vaccination coverage statistics. Objective: To describe the coverage of recommended vaccines during the pre-pandemic (2015-2019) and pandemic (2020-2021) periods in Paraguay. Materials and Methods: This was a descriptive, observational crosssectional study, comprising children from 0 to 5 years of age, from the Country's 18 Health Regions. The vaccination coverage of the National Expanded Immunization Program, pre-pandemic (2015-2019) and pandemic (2020-201) periods were analyzed. The recommended vaccinations included were: BCG, DTP1, DTP3, IPV1 and bOPV3, MMR1, MMR2 and yellow fever vaccine (AA), the calculation of vaccination coverage at the country level was carried out through the analysis of the weekly electronic report of doses of vaccines administered by Health Region. The comparison of vaccination coverage by recommended vaccine was established, by periods and age range. Results: During the pandemic, a decrease in vaccination coverage of the recommended vaccines was observed, for BCG: $4 \%$ (2020), 15\% (2021);DTP1: $5 \%$ (2020), 13\% (2021); DTP3: 9\% (2020), 22\% (2021); IPV1: 5\% (2020), 16\% (2021); bOPV3: 7\% (2020), 19\% (2021); MMR1: 9\%, MMR2: 13\% (2020), MMR1: 17\%, MMR2: 16\% (2021) and AA: $7 \%$ (2020), 15\% (2021). Conclusion: During the Covid19 pandemic, vaccination coverage of all recommended vaccines decreased, a similar decrease was found in other countries in the Americas, there is a risk of outbreaks of

Correspondencia: Soraya Araya, correo: angelaarayayampey@gmail.com

Conflicto de interés: los autores declaran no poseer conflicto de interés, se aclara que ninguno trabaja para laboratorios fabricantes de vacunas.

Fuente de financiamiento: autofinanciado

Recibido: 25/10/2021 Aceptado: 19/11/2021

DOI: https://doi.org/10.31698/ped.48032021003

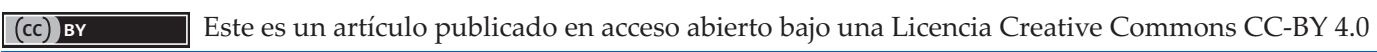


brotes de enfermedades prevenibles por vacunación por el acúmulo de susceptibles.

Palabras Claves: Cobertura vacunal, pandemia, programa deinmunización, COVID-19.

\section{INTRODUCCIÓN}

El virus SARS-CoV-2 fue identificado por primera vez en diciembre de 2019 en la ciudad de WuhanChina, dicho agente es el causante de una enfermedad respiratoria denominada COVID-19, cuyo grado de severidad es variable, de formas sintomáticas leves a graves, pudiendo incluso causar la muerte de adultos mayores y personas con enfermedad de base, si bien la severidad es menor en niños, lacomplicación denominada síndrome inflamatorio multisistémico asociado a COVID-19 posee una tasa de letalidad del 6,4 \% en Paraguay, según datos reportados por la Dirección General de Vigilancia de la Saluddel Ministerio de Salud Pública y Bienestar Social ${ }^{(1,2)}$.

El nuevo coronavirus se expandió rápidamente por todo el mundo, dando lugar a una de las mayores pandemias registradas en la historia.El primer caso en las Américas fue notificado en enero del año 2020, al 24 de agosto del 2021, el 39\% de los casos notificados y el $47 \%$ de las muertes registradas, correspondieron al Continente Americano. Cuatro países: Argentina, Brasil, Colombia y Estados Unidos de América, se ubicaron entre los 10 países que notificaron el mayor número de casos acumulados a nivel mundial, mientras que cinco países: Brasil, Colombia, Estados Unidos de América, México y Perú, se ubicaron entre los 10 países con el mayor número de muertes acumuladas ${ }^{(3)}$.

A setiembre del año 2021, la región de las Américas continuó siendo la más afectada, con 88.207.746 casos confirmados y 2.175 .310 muertes $^{(3)}$.

En Paraguay el primer caso de COVID-19 fue confirmado el 7 de marzo del año 2020; tras el análisis de la situación epidemiológica mundial y la valoración del comportamiento agresivo del virus en otros países tales como Estados Unidos, España, vaccine-preventable diseases due to the accumulation of susceptible populations.

Keywords: Vaccine coverage, pandemic, immunization program, COVID-19.

Francia e Italia, el 10 de marzo del año 2020 se ordenó el cierre de escuelas por medio del Decreto Presidencial y se instauraron medidas sanitarias de restricción de la circulación y distanciamiento social con el objetivo de mantener plana la curva epidemiológica hasta que se fortaleciera el sistema de salud, surgiera alguna vacuna eficaz y segura o algún medicamento que propiciara la cura; semejantes acciones fueron implementadas por varios países en todo el mundo ${ }^{(4)}$.

Durante la Pandemia se generaron factores que afectaron el funcionamiento de los programas regulares de vacunación, tales como el cierre de fronteras, restricción de vuelos que en algunos países dió lugar a la recepción tardía de vacunas, por otro lado la redistribución de enfermeros/as de áreas de vacunación a áreas de atención de pacientes con sospecha de Covid-19 disminuyó el recurso humano de los vacunatorios, el miedo al contagio y las medidas de restricción de circulación disminuyeron notablemente la afluencia de personas a los vacunatorios, afectando las coberturas de vacunación en varios países ${ }^{(5)}$.

En los principios rectores de la Organización Mundial de la Salud para las actividades de vacunación durante la Pandemia de Covid-19, se realizó un llamado a los países a mantener los servicios de vacunación programática funcionantes, mientras se pudiera garantizar condiciones seguras frente a la circulación de SARS-CoV-2, fue así que en mayo del año 2020 se implementó en Paraguay el Consenso de Vacunación en tiempos de Pandemia de COVID-19: Acciones para la sostenibilidad del Programa Ampliado de Inmunización (PAI), elaborado por el Comité Técnico Asesor de Inmunizaciones y el equipo Técnico del Programa Ampliado de Inmunización, con el objetivo de evitar 
la disrupción del programa de vacunación y aparición de brotes de enfermedades prevenibles por vacunas, las que implicarían una carga adicional al ya exigido sistema de salud ${ }^{(6,7)}$.

El objetivo del presente estudio es describir la cobertura de vacunación de los biológicos trazadores del Calendario Regular de Vacunación del Paraguay durante los años 2020 y 2021 en el contexto de la pandemia por SARS-CoV-2 respecto del periodo prepandémicos: 2015-2019.

\section{MATERIALES Y MÉTODOS}

Estudio observacional, descriptivo, de corte transverso, llevado a cabo por el Programa Ampliado de Inmunización del Paraguay, se valoraron las coberturas de vacunación en niños y niñas con edad comprendida 0 días a 5 años de edad, de las 18 Regiones Sanitarias que conforman el país. Se analizaron las coberturas de vacunación de los biológicos trazadores durante el periodo prepandémico (años 2015 a 2019) y pandémico (años 2020 a 2021).

\section{DEFINICIÓN DE VARIABLES:}

Biológicos trazadores: fueron consideradas las siguientes vacunas: vacuna BCG, vacuna DPT primera dosis y tercera dosis (si bien en Paraguay se administra la vacuna pentavalente, teniendo en cuenta que el indicador mundial es DPT, la cual está contenida en la vacuna pentavalente, para el presente artículo hacemos referencia a DPT con el fin de unificar criterio al momento de establecer comparación con las coberturas de vacunación de otros países, del reporte WUENIC de la Organización Mundial de la Salud. ${ }^{8}$ ), vacuna IPV1 y bOPV3, vacuna contra el Sarampión SPR primera y segunda dosis y vacuna antiamarílica (FA).

Cobertura anual de vacunación: Para el cálculo de cobertura anual de vacunación por biológico trazador a nivel país, se analizaron la cantidad de dosis administradas por vacuna según la edad objetivo de la misma, reportadas semanalmente por las regiones sanitarias hasta la semana epidemiológica 52, a través de la planilla electrónica PAI VISUAL, utilizando como denominador la estimación y proyección de población por edad para dicho año del INE (Instituto Nacional de Estadística), de esta manera se procedió para los años 2015 a 2020. Para el año 2021, se realizó la proyección estimada de cobertura a la semana epidemiológica 52, en base a las coberturas alcanzadas a la semana epidemiológica 35.

Se detalla a continuación el Calendario Regular de Vacunación del Paraguay, año 2021 (ver Figura 1):

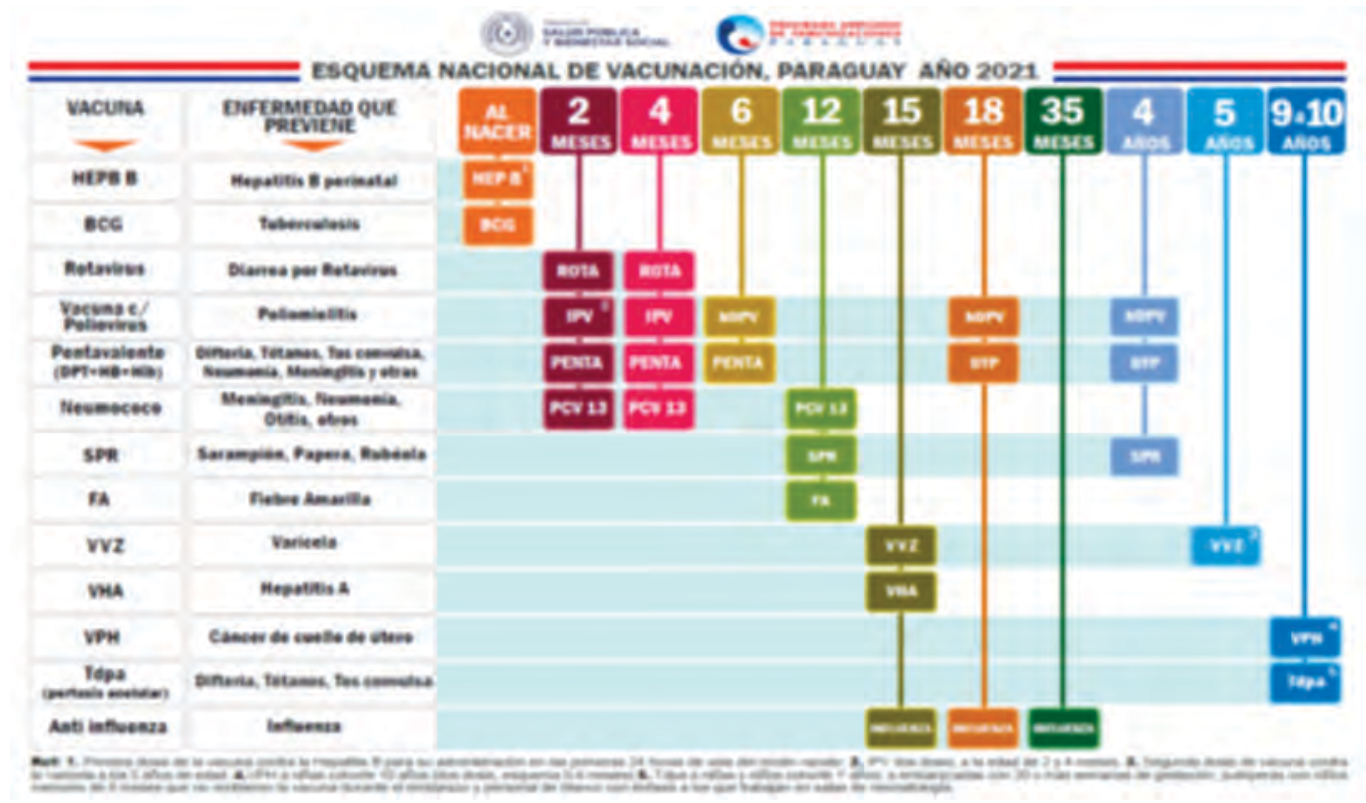

Figura 1. Calendario Regular de Vacunación, Paraguay Año 2021 
Las coberturas de vacunación de los biológicos trazadores fueron analizados por año, por periodo:prepandémico (2015 a 2019) y pandémico (2020-2021), como así también por grupos de edad, se realizaron análisis estadísticos.

\section{RESULTADOS}

Durante el periodo prepandémico (años 2015 a 2019) no se alcanzó la cobertura ideal de vacunación del 95 $\%$ en ningún biológico trazador, oscilando las coberturas de vacunación entre un 60\% y $91 \%$ (ver Tabla 1).

Al establecer la comparación de coberturas periodo prepandémico y pandémico, se constató un descenso de la cobertura de vacunación en todos los biológicos trazadores durante el periodo pandémico (ver Tabla 2).

Al enfocarnos en los años pandémicos 2020-2021, respecto al año 2019, se registró un descenso para BCG $4 \%$ (año 2020) y $15 \%$ (año 2021), DPT1: 5 \% (año
2020) y $13 \%$ (año 2021), para DPT3: 9 \% (año 2020) y $22 \%$ (año 2021), el descenso de cobertura para la vacuna inactivada contra la poliomielitis IPV1 fue del $5 \%$ (año 2020) y $16 \%$ (año 2021), mientras que para tercera dosis de la vacuna contra la poliomielitis (bOPV3) el descenso fue de $7 \%$ (año 2020) y $19 \%$ (año 2021) (ver Tabla 2).

Respecto a la vacunación contra el Sarampión, el descenso constatado fue del $9 \%$ para primera dosis y $13 \%$ para segunda dosis para el año 2020, mientras que el descenso durante el año 2021 fue de 17\% para primera dosis y $16 \%$ para segunda dosis. (ver Tabla 2 ).

Finalmente, al analizar las coberturas de vacunación durante los años 2020 y 2021 respecto al año 2019, por grupos de edad: menores de 1 año, 12 a 18 meses, 4 a 5 años, se constató que durante el año 2020el mayor descenso de coberturas de vacunación se registró en el grupo de niños de 4 a 5 años, mientras que al siguiente año el mayor descenso se observó en el grupo de niños menores de 1 año de edad (Tabla 3).

Tabla 1. Coberturas Anuales de Vacunación Biológicos Trazadores Año 2015-2021.

\begin{tabular}{lccccccc}
\hline Vacuna & $\begin{array}{c}\text { Cobertura } \\
\text { Año 2015 }\end{array}$ & $\begin{array}{c}\text { Cobertura } \\
\text { Año 2016 }\end{array}$ & $\begin{array}{c}\text { Cobertura } \\
\text { Año 2017 }\end{array}$ & $\begin{array}{c}\text { Cobertura } \\
\text { Año 2018 }\end{array}$ & $\begin{array}{c}\text { Cobertura } \\
\text { Año 2019 }\end{array}$ & $\begin{array}{c}\text { Cobertura } \\
\text { Año 2020 }\end{array}$ & $\begin{array}{c}\text { P. Cobertura } \\
\text { Año 2021* }\end{array}$ \\
\hline BCG & 83 & 82 & 84 & 79 & 75 & 71 & 60 \\
\hline IPV1 & $0,2^{* *}$ & 82 & 83 & 80 & 76 & 71 & 60 \\
\hline DPT1 & 84 & 82 & 83 & 80 & 76 & 71 & 63 \\
\hline DPT3 & 80 & 80 & 79 & 76 & 74 & 65 & 52 \\
\hline bOPV3 & 80 & 80 & 79 & 75 & 71 & 64 & 52 \\
\hline SPR1 & 66 & 91 & 80 & 81 & 75 & 66 & 58 \\
\hline FA & 71 & 80 & 79 & 80 & 70 & 63 & 55 \\
\hline SPR2 & 60 & 75 & 70 & 71 & 71 & 58 & 55 \\
\hline
\end{tabular}

* Cobertura Proyectada a la SE52

$* *$ Switch bOPV/IPV

Tabla 2. Coberturas de vacunación de los biológicos trazadores, años 2020 y 2021 en relación al año 2019

\begin{tabular}{lcccccc}
\hline Vacuna & $\begin{array}{c}\text { Cobertura } \\
\text { Año 2019 }\end{array}$ & $\begin{array}{c}\text { Cobertura } \\
\text { Año 2020 }\end{array}$ & $\begin{array}{c}\% \\
\text { Descenso }\end{array}$ & $\begin{array}{c}\text { Cobertura SE 35 } \\
\text { Año 2021 }\end{array}$ & $\begin{array}{c}\text { Cobertura Proyectada } \\
\text { Año2021* }\end{array}$ & $\begin{array}{c}\% \\
\text { Descenso }\end{array}$ \\
\hline BCG & 75 & 71 & 4 & 41 & 60 & 15 \\
\hline IPV1 & 76 & 71 & 5 & 41 & 60 & 16 \\
\hline DPT1 & 76 & 71 & 5 & 42 & 53 & 22 \\
\hline DPT3 & 74 & 65 & 9 & 35 & 52 & 13 \\
\hline bOPV3 & 71 & 64 & 7 & 35 & 58 & 17 \\
\hline SPR1 & 75 & 66 & 9 & 39 & 55 & 15 \\
\hline FA & 70 & 63 & 7 & 37 & 37 & 16 \\
\hline SPR2 & 71 & 58 & 13 & 37 & & 16 \\
\hline
\end{tabular}

* Proyección de cobertura a la SE 52 en base a la SE35, año 2021 
Tabla 3. Cobertura de Vacunación por grupo de edad años 2020-2021, en relación al año 2019 (prepandémico)

\begin{tabular}{lcc}
\hline Edad & $\begin{array}{c}\text { \% Descenso } \\
\text { Año 2020 }\end{array}$ & $\begin{array}{c}\text { Descenso estimado } \\
\text { Año 2021 }\end{array}$ \\
\hline$<1$ año & $4-7$ & $13-22$ \\
\hline 12 a 18 meses & $7-9$ & $15-17$ \\
\hline $4-5$ años & 13 & 16 \\
\hline
\end{tabular}

\section{DISCUSIÓN}

El equilibrio entre la respuesta a la Pandemia y las demás necesidades del sistema de salud fue afectado en todo el mundo, impactando en los programas de vacunación en varios países y continentes, tal es así, que la encuesta sobre servicios de salud esenciales durante la Pandemia de SARS-CoV-2 realizada por la OMS, halló que de 105 países participantes, 90\% reportó interrupción de los servicios de salud, siendo el servicio esencial más afectado el de la vacunación programática y extramural $(70 \%)$, seguido de diagnóstico y tratamiento de enfermedades no transmisibles (69\%), en relación a este punto cabe destacar que el Paraguay logró mantener el funcionamiento del Programa Ampliado de Inmunizaciones a través de los servicios vacunatorios sin la disrupción del mismo durante los años pandémicos 2020 y $2021^{(5,7)}$.

En cuanto a las coberturas de vacunación de los biológicos trazadores en nuestro país, durante el año 2020 se constató un descenso en las coberturas de vacunación respecto al año 2019 de 4 a 13 \%, siendo mayormente afectado el grupo de niños de 4 a 5 años, no obstante al siguiente año el descenso de coberturas de vacunación fue 13 a $22 \%$ respecto al año 2019, siendo afectados en mayor medida los niños menores de 1 año, el mayor descenso en el año 2021 en relación al año 2020 podría deberse al aumento de la percepción de riesgo por parte de la ciudadanía, tras el aumento del índice de contagio de COVID-19evidenciado por el aumento de la curva epidemiológica durante el primer y segundo trimestre del año $2021^{(2,8)}$.

En cuanto a la Situación de las Coberturas de Vacunación de los Biológicos Trazadores del Paraguay respecto a otros países de América Latina se ha constado similar comportamiento de las coberturas de vacunación durante la pandemia, tal es así que la cobertura de vacunación de BCG tuvo un descenso significativo en México, Brasil, Argentina, Bolivia, Venezuela, manteniendo la cobertura Colombia, Chile y Costa Rica ${ }^{(9,10)}$. En India se registró un descenso del $52 \%$ en el promedio diario de vacunaciones, siendo el grupo más afectado el de recién nacidos con un descenso en la cobertura de BCG del $40,6 \%{ }^{(10)}$. En cuanto a vacuna DPT1 las coberturas se mantuvieron estables para Chile, Costa Rica y Uruguay, para DPT3 se mantuvo en Costa Rica, viéndose disminuida en Venezuela, Bolivia, Argentina, México, Brasil, Paraguay y Colombia, por otra parte en Europa, la caída de coberturas de vacunación para este biológico trazador fue del $35 \%$ en Francia y 6,7\%en Reino Unido ${ }^{(9-12)}$.

Teniendo en cuenta la circulación de virus de Sarampión durante la pandemia de SARS-CoV-2en varios países de América Latina y Europa, resulta relevante el comportamiento de la cobertura de vacunación de la vacuna SPR; en nuestro paísel descenso de cobertura SPR1fue 9\% (año 2020) y $17 \%$ (año 2021), en México, Brasil, Argentina, Bolivia y Venezuela también se constataron descensos, manteniéndose las coberturas de vacunación en Chile, Costa Rica y Uruguay ${ }^{(8)}$. Por otra parte, en Europa: la cobertura de vacunación SPR1 descendió $30 \%$ en España y $43 \%$ en Francia ${ }^{(11-13)}$.

En el sureste de Asia la cobertura de vacunación descendió 7,4\% para SPR1, mientras que en países de alta economía como Estados Unidos el descenso de cobertura de vacunación para SPR1 fue aún mayor siendo del 20,5\% al mes de abril ${ }^{(12-14)}$.

En cuanto a la cobertura de vacunación SPR2 la misma se mantuvo estable en Costa Rica y Colombia, 
constatándose un descenso mayor al de Paraguay en Venezuela, Brasil, Bolivia y Argentina ${ }^{(8,9,11)}$.

La situación global de bajas coberturas ha generado un aumento de la población susceptible a enfermedades infecciosas prevenibles por vacunación, al punto que al finalizar el año 2020, 30 millones de niños no recibieron la tercera dosis de DPT y 27,2 millones de niños no recibieron la primera dosis de la vacuna antisarampionosa (SPR), lo cual establece las condiciones para brotes de sarampión, difteria y coqueluche ${ }^{(5)}$. Si bien constituye una buena práctica de los Programas de Inmunización la realización de una Campaña Nacional de Vacunación cuando la población susceptible al sarampión o poliomielitis alcanza cifras iguales o mayores al de una cohorte de recién nacidos, durante el año 2020 la Organización Mundial de la Salud recomendó a los países la suspensión temporal de las Campañas Nacionales de Vacunación contra el Sarampión y la Poliomielitis, con el fin de reducir la transmisión comunitaria del SARS-CoV-2 y fueron suspendidas 48 campañas de vacunación contra la Poliomielitis y 37 campañas de vacunación contra el Sarampión. Consecuentemente se reportaron casos de poliovirus derivado de la vacuna en más de 30 países, brotes de poliomielitis en Nigeria, Pakistán y Afganistán, además de brotes de Sarampión en 18 países en el año 2020. Durante el año 2021 hubo brotes de Sarampión en Brasil y Estados Unidos ${ }^{(15-17)}$.

El descenso de coberturas de vacunación de los biológicos trazadores registrado en Paraguay durante la presente pandemia, junto a las coberturas de vacunación inferiores al $95 \%$ en los últimos años,además de losbrotes desarampión, difteria y poliomielitis en otros países, sitúa al país en una situación de riesgo para la aparición de enfermedades prevenibles por vacunación tras el cese de las medidas de restricción de circulación instauradas ante el SARS-CoV-2.

Aspectos éticos: se respetó la confidencialidad de los datos de filiación.

\section{CONCLUSIÓN}

Durante los años 2020 y 2021, la Pandemia Covid-19 generó un descenso de las coberturas de vacunación de los biológicos trazadores del calendario regular de vacunación del Paraguay, mismo fenómeno se observó en otros países de la Región.

Urge la realización de la Campaña Nacional de Vacunación contra la Poliomielitis y el Sarampión con el fin de mantener la certificación de país libre de Sarampión, como así también la puesta en marcha de la recuperación de esquemas atrasados de vacunación de las demás vacunas programáticas.

\section{CONTRIBUCIÓN DE AUTORÍA:}

Soraya Araya: Concepción y diseño de la idea, elaboración del protocolo, recolección de datos, análisis de datos, elaboración de resultados, elaboración de la discusión, revisión de la bibliografía, redacción del manuscrito y revisión del trabajo realizado por el equipo.

Teresa Pérez: Colaboración en la recolección de la información, redacción de materiales y métodos.

Avelina Troche: Colaboración en la elaboración del resumen, colaboración en la discusión.

Julio Nissen: Colaboración en el análisis de datos.

Luis Cousirat: Colaboración en la recolección de datos y elaboración de tablas

Leticia Nuñez: Colaboración en la elaboración de tablas

Diego Revolero: Colaboración en la recolección de datos, colaboración con los gráficos.

Iván Salas: Elaboración de gráficos.

Silvia Battaglia: Elaboración del resumen

Martha Von Horoch: Colaboración en la búsqueda bibliográfica

Héctor Castro: Colaboración en la búsqueda bibliográfica, autorización para la elaboración del trabajo de investigación. 


\section{REFERENCIAS}

1. Huang C, Wang Y, Li X, Ren L, Zhao J, Hu Y, et al. Clinical features of patients infected with 2019 novel coronavirus in Wuhan, China. Lancet. 2020; 395(10223):497-506. doi: https://doi.org/10.1016/S0140-6736(20)30183-5

2. Dirección General de Vigilancia de la Salud Paraguay. Reporte $\mathrm{N}^{\circ} 139$ [Internet] 2021 [Citado 2021 oct 10] Disponible en: https://dgvs.mspbs.gov.py/files/ boletines_covid19/SE45_2021_Boletin_Covid19.pdf

3. COVID-19. Respuesta de la OPS/OMS Reporte 58 (27 de agosto del 2021). [Internet] 2021 [Citado 2021 oct 10]. Disponible en: https://www.paho.org/es/documentos/ covid-19-respuesta-opsoms-reporte-58-27-agosto-2021

4. Ministerio de Salud Pública y Bienestar Social, Dirección de Vigilancia de la Salud. COVID-19. [Sitio web] 2021 [Citado 2021 oct 10]. MSPyBS. Disponible en: https://dgvs.mspbs.gov.py/views/paginas/covid19.html

5. WHO. World Health Organization in Who global pulse survey, $90 \%$, of countries report disruptions to essential health services since COVID-19 pandemic 2020. [Internet] 2020 [Citado 2021 oct 10] Available from: https://www.wh o.int/news/item/31-08-2020-in-who-global-pulse-survey90-of-countries-report-disruptions-to-essencial-healthservice-since-covid-19-pandemic

6. OMS. Principios rectores para las actividades de inmunización durante la pandemia de COVID-19: orientaciones provisionales, 26 de marzo de 2020. [Internet] 2020 [Citado 2021 oct 10] Available from: http://apps.who.int/iris/bitstream/handle/10665/331670/ WHO-2019-nCoV-immunization_services-2020.1spa.pdf? sequence $=1$ \&isAllowed $=y$

7. Ministerio de Salud Pública y Bienestar Social. Consenso de Vacunación en tiempos de Pandemia de COVID-19, acciones para la sostenibilidad del Programa Ampliado de Inmunizaciones del Paraguay. Resolución DG № 389 .

8. WHO. WHO/UNICEF estimates of national immunization coverage. WUENIC excel. [Internet] 2021 [Citado 2021 oct 10] Available from: https://www.who.int/teams/immunization-vaccines-andbiologicals/immunization-analysis-and-insights/globalmonitoring/immunization-coverage/who-unicefestimates-of-national-immunization-coverage

9. Torner N. Collateral effects of COVID-19 pandemic emergency response on worldwide inmunizations. Vacunas. 2020; 21(2):73:5. doi: https://doi.org/10.1016/ j.vacun.2020.07.002
10. Bastías M, Brstilo I, González C. Vacunación programática 2020 en Chile en tiempos de pandemia por SARS-CoV-2. Rev Chilena Infectol 2021; 38(3):335-361. doi:http://dx.doi.org/10.4067/S0716-10182021000300355

11. Cause K, Fullman N, Sorensen RJD, Galles NC. Estimating global and regional disruptions to routine childhood vaccine coverage during the COVID-19 pandemic in 2020: a modeling study. Lancet 2021; 398:52234. doi: https://doi.org/10.1016/S0140-6736(21)01337-4

12. McDonald HI, Tessier E, White JM, Woodruff M, Knowles C, Bates C, et al. Early impact of the coronavirus disease (COVID-19) pandemic and physical distancing measures on routine childhood vaccinations in England, January to April 2020. Euro Surveill. 2020; 25(19):2000848. doi: https://doi.org/10.2807/1560-7917.ES.2020.25.19.2000848

13. Dinleyici EC, Borrow R, Safadi MAP, van Damme P, Munoz FM. Vaccines and routine immunization strategies during the COVID-19 pandemic. Hum Vaccin Immunother. 2021; 17(2):400-407. doi: https://doi.org/10. $1080 / 21645515.2020 .1804776$

14. Santoli JM, Lindley MC, De Silva MB, Kharbanda EO, Daley MF, Galloway L. Effects of the COVID-19 pandemic on routine pediatric vaccine ordering and administration United States, 2020. MMWR Morb Mortal Wkly Rep. 2020; 69:591-593. doi: https://doi.org/10.15585/mmwr.mm6919e2

15. WHO. Niger reports new polio outbreak. [Internet] 2021 [Citado 2020 Jun 29]. https://www.who.int/immuni zation/diseases/measles/statement_missing_measles_vac cines_covid-19/en/

16.Bramer CA, Kimmins LM, Swanson R, Kuo J, Vranesich $\mathrm{P}$, Jacques-Carroll LA, et al. Decline in child vaccination coverage during the COVID-19 pandemic - michigan care improvement registry, May 2016-May 2020. MMWR Morb Mortal Wkly Rep. 2020; 69(20):630-31. doi: https://doi.org /10.15585/mmwr.mm6920e1

17. OPS/OMS. Actualización Epidemiológica: Sarampión (4 de octubre de 2021). [Internet] 2021 [Citado 2020 Jun 29] Available from: https://www.paho.org/es/documentos/ actualizacion-epidemiologica-sarampion-4-octubre-2021 